\title{
Breakup of a viscous liquid jet in a surrounding gas flowing through a coaxial cylindrical sheath
}

\author{
Takao YOSHINAGA \\ Research Center for Highly-Functional Nanoparticles, Doshisha University, Kyoto, Japan \\ Corresponding author email: tyoshina@mail.doshisha.ac.jp
}

\begin{abstract}
Breakup phenomena of a viscous liquid column jet in an inviscid stationary surrounding gas are analytically investigated, when the jet flows through a coaxial cylindrical sheath. Under a long wave approximation, axisymmetric nonlinear evolution equations of the jet and surrounding gas are derived and numerically solved under a spatially periodic boundary condition. Validity and limitation of the approximation are examined in the linear regime in comparison with exact instabilities. It is shown in the nonlinear analysis that there exist three types of breakup modes: the Rayleigh mode in which the surface tension instability is dominant and the breakup is caused by pinching, the first wind-induced mode in which blobs connected by threads are produced by increased aerodynamic instability and the second wind-induced mode in which the jet surface deforms to be spiked like a cusp without pinching and droplets are to be produced from a tip of the cusp. Existing regions of these modes are examined in the parameter space of the Weber number and the sheath radius for different Reynolds numbers. As a consequence, the closely placed sheath wall always suppresses the breakup by pinching and results in the appearance of the second wind-induced mode with cusp profiles.
\end{abstract}

\section{Keywords}

liquid jet, surrounding gas, nonlinear, instability, breakup

\section{Introduction}

Liquid jets are of great importance in engineering and industrial applications in producing microdrops and thin fibers, where destabilization in the former and stabilization in the latter play an important role. Such instabilities have been investigated experimentally and theoretically [1, 2, $3,4,5]$ since Lord Rayleigh [6] examined linear instabilities of periodic disturbances on a liquid column.

It is now experimentally known that, when the jet is emanating from a circular nozzle in a surrounding gas, liquid drops are naturally produced from the jet with or without satellites after breaking up due to the instabilities $[7,8,9,10]$. In a laminar jet, the surface tension dominant (Rayleigh) mode mainly appears in a low emanating speed, while in a high emanating speed the Taylor mode dominates due to the aerodynamic instability, which consists of the first wind-induced and the second wind-induced modes. As the flow speed increases, a transition appears from the first to the second wind-induced mode, where blobs connected by threads are produced in the former, while the jet surface deforms to be spiked like a cusp without pinching and droplets are to be produced from a tip of the cusp in the latter.

Theoretically, the Rayleigh mode has been examined in both linear and nonlinear analyses and the mechanism of producing liquid drops and satellites is rather well understood (for example, $[5,4]$ and refer the references therein). However, the mechanism of producing fine droplets in the Taylor mode, particularly the second wind-induced mode, is not sufficiently understood even now. Most of the analyses are made in the linear regime and show that fine liquid drops are produced due to not only the shift of a lower unstable wave number region to a higher region, but also the appearance of the unstable asymmetric modes [11, 12, 13].

In the nonlinear analysis, on the other hand, Spangler et al. [14] numerically examined the axisymmetric deformations of the jet at a medium speed which is typical in the Taylor mode 
regime when the jet and surrounding gas are assumed to be the potential flows. It is shown in their results that the jet surface deforms to be spiked like a cusp in the second-wind induced mode. They expected that a liquid ring is produced around the jet from the tip of the cusp and later splits into a series of droplets circumferentially. This scenario of producing droplets in the second wind-induced mode is possible even for axisymmetric disturbances at such a flow speed that the asymmetric modes do not yet grow large.

In the above analyses the surrounding gas phase is assumed to be unboundedly extended. In practice, however, the surrounding gas would be usually bounded by a solid wall like a duct with various cross sections or influenced by any other constructions such as an experimental set up, whether they affect behavior of the jet or not. From this point of view, it is important to understand the influence of such external boundaries on the breakup of the jet, particularly, in a high speed jet where motions of the surrounding gas play an important role. In the present paper, as the simplest case of the external boundaries, considered is a coaxial cylindrical sheath through which a viscous jet in a surrounding gas is flowing. There may exist a critical sheath radius for which the sheath wall significantly affects the aerodynamic instability, since the influence of the sheath on the aerodynamic instability diminishes as the radius of the sheath becomes larger. In this sense, the present problem is different from usual two-layer coaxial flows in a pipe. The end of this study is to theoretically clarify the influence of a coaxial sheath wall on the aerodynamic instability. The analysis is performed in order to understand influence of the sheath wall on the linear and nonlinear instabilities of the jet which results from the surface tension, velocity and density differences and gap length.

\section{Reduced long wave equations}

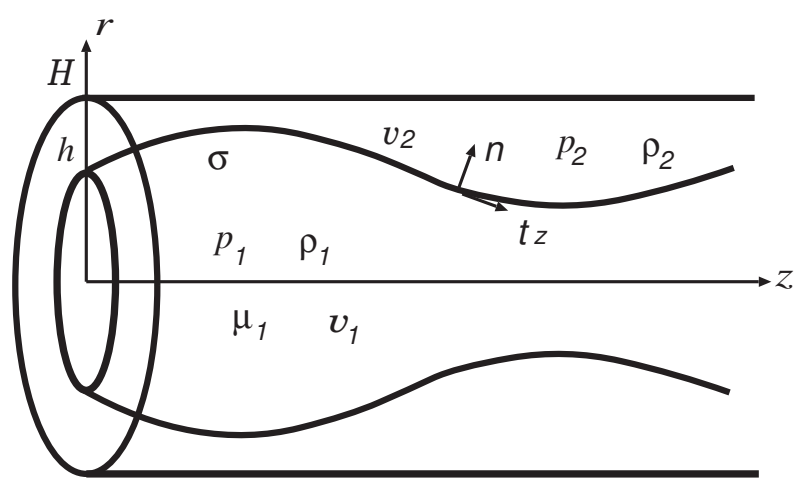

Figure 1. Schematic of a liquid jet in a surrounding gas flowing through a coaxial cylindrical sheath.

As is shown in Fig. 1, we consider a problem that a liquid jet of radius $r=h(z, t)$ flows in a surrounding gas through a coaxial cylindrical sheath of the constant radius $r=H(h<H)$ in the $(r, z)$ axisymmetric cylindrical coordinate system and time $t$. Denoting the jet and the surrounding gas as $j=1$ and 2 , respectively, the velocity vector $\boldsymbol{v}_{j}=\left(u_{j}, w_{j}\right)$, the pressures $p_{j}$ are functions of $r, z$ and $t$. The surface tension is denoted as $\sigma$, the densities as $\rho_{j}$ and the viscosities as $\mu_{j}$. For simplicity, the viscosity of the surrounding gas is neglected $\left(\mu_{2}=0\right)$ and the gravitational effect is ignored since a sufficiently large velocity flow is considered in a quiescent gas medium. In addition, both jet liquid and surrounding gas are assumed to be incompressible. The outward normal unit vector on the jet surface is denoted as $n$, while the surface tangential unit vector as $t_{z}$ in the flow direction. The basic equations consist of the continuity and momentum equations, where these are of the jet $(j=1)$ for $0 \leq r<h$ and of the surrounding gas $(j=2)$ for $h<r<H$. On the other hand, the boundary conditions consist of the well-known kinematical and dynamical conditions on the jet surface at $r=h(z, t)$, while at $r=H$ only imposed is the condition of $u_{2}=0$.

Then, the following expansions with respect to the jet radius and the surrounding gas phase thickness are introduced [15]: 
(i) liquid jet $(0 \leq r<h)$ :

$$
\begin{aligned}
& w_{1}=w_{1}^{(0)}+r^{2} w_{1}^{(2)}+\cdots, \\
& p_{1}=p_{1}^{(0)}+r^{2} p_{1}^{(2)}+\cdots,
\end{aligned}
$$

(ii) surrounding gas $(h<r<H)$ :

$$
\begin{aligned}
& u_{2}=u_{2}^{(0)}+(r-R) u_{2}^{(1)}+(r-R)^{2} u_{2}^{(2)}+\cdots, \\
& w_{2}=w_{2}^{(0)}+(r-R) w_{2}^{(1)}+(r-R)^{2} w_{2}^{(2)}+\cdots, \\
& p_{2}=p_{2}^{(0)}+(r-R) p_{2}^{(1)}+(r-R)^{2} p_{2}^{(2)}+\cdots,
\end{aligned}
$$

where the radius at the mid-plane of the surrounding gas phase $R \equiv(H+h) / 2$ is introduced. In the above representations, the dependent variables $w_{1}, p_{1}, u_{2}, w_{2}$ and $p_{2}$ are functions of $r, z$ and $t$, while $R$ and coefficients of the expansions are functions of $z$ and $t$, although $u_{1}$ can be obtained through the equation of continuity.

Substituting the above expansions (1) and (2) into the basic equations and the boundary conditions, we retain the lowest order terms under the following conditions: the order of magnitude of variables is assumed to be $h \lesssim b \ll \lambda$ when $b \equiv H-h$ is the gas phase thickness and $\lambda$ is the characteristic wave length of deformations, where $H$ and $\lambda$ are taken to be of $O(1)$. Then, the magnitude of the expansion parameters is $r^{2} \leq h^{2}=O\left(h^{2}\right)$ in (1) and $|r-R| \leq b / 2=O(b)$ in (2), while $R=O(1)$ and $\partial / \partial z=O(1)$ for $z \sim \lambda$, in addition to $\partial / \partial t=O(1)$.

As a result of this, we finally obtain the following nonlinear simultaneous equations in the nondimensional forms [15]:

$$
\begin{aligned}
\frac{\partial h}{\partial t} & =-w_{1} \frac{\partial h}{\partial z}-\frac{h}{2} \frac{\partial w_{1}}{\partial z}, \\
\frac{\partial w_{1}}{\partial t} & =-w_{1} \frac{\partial w_{1}}{\partial z}-\frac{\partial p_{1}}{\partial z}+\frac{1}{\operatorname{Re}}\left(2 \frac{\partial^{2} w_{1}}{\partial z^{2}}+\frac{6}{h} \frac{\partial h}{\partial z} \frac{\partial w_{1}}{\partial z}\right), \\
\frac{\partial w_{2}}{\partial t} & =-w_{2} \frac{\partial w_{2}}{\partial z}-\frac{1}{\gamma}\left(\frac{\partial p_{2}^{(0)}}{\partial z}-p_{2}^{(1)} \frac{\partial R}{\partial z}\right), \\
\frac{\partial u_{2}}{\partial t} & =-w_{2} \frac{\partial u_{2}}{\partial z}-\frac{1}{\gamma} p_{2}^{(1)},
\end{aligned}
$$

where the superscripts on $w_{1}, w_{2}, u_{2}$ and $p_{1}$ have been omitted. In the above equations, the gas pressures $p_{2}^{(0)}$ and $p_{2}^{(1)}$ are governed by the following equations in each time through the pressure relation between the core and gas $p_{1}=p_{2}^{(0)}+p_{2}^{(1)}(h-R)+\frac{1}{\mathrm{~Wb}} \kappa-\frac{1}{\operatorname{Re}} \frac{\partial w_{1}}{\partial z}$ :

$$
\begin{aligned}
& \left(A_{1} \frac{\partial^{2}}{\partial z^{2}}+A_{2} \frac{\partial}{\partial z}\right) p_{2}^{(0)}+\left(A_{3} \frac{\partial^{2}}{\partial z^{2}}+A_{4} \frac{\partial}{\partial z}+A_{5}\right) p_{2}^{(1)}+A_{6}=0, \\
& \left(B_{1} \frac{\partial^{2}}{\partial z^{2}}+B_{2} \frac{\partial}{\partial z}\right) p_{2}^{(0)}+\left(B_{3} \frac{\partial^{2}}{\partial z^{2}}+B_{4} \frac{\partial}{\partial z}+B_{5}\right) p_{2}^{(1)}+B_{6}=0,
\end{aligned}
$$

where the coefficients $A_{1}$ to $A_{6}$ and $B_{1}$ to $B_{6}$ are rather complicated functions of $h, w_{1}, w_{2}$ and $u_{2}$. In the representations (3) to (7), the length and velocity have been nondimensionalized by the undisturbed jet radius $a$ and undisturbed axial velocity of the jet $W$ and, so that, the time is nondimensionalized by $a / W$, while the pressure by $\rho_{1} W^{2}$. Then the following nondimensional parameters are introduced: the Weber number $\mathrm{Wb}=\rho_{1} W^{2} a / \sigma$, the Reynolds number $\mathrm{Re}=$ $\rho_{1} W a / \mu_{1}$, the density ratio $\gamma=\rho_{2} / \rho_{1}$ and the sheath radius $H$.

\section{Validity and limitations of the approximation}

Before going on to the nonlinear analysis based on the preceding reduced equations, it is beneficial to examined the linear instabilities and to know validity and limitation of the present 
(a)

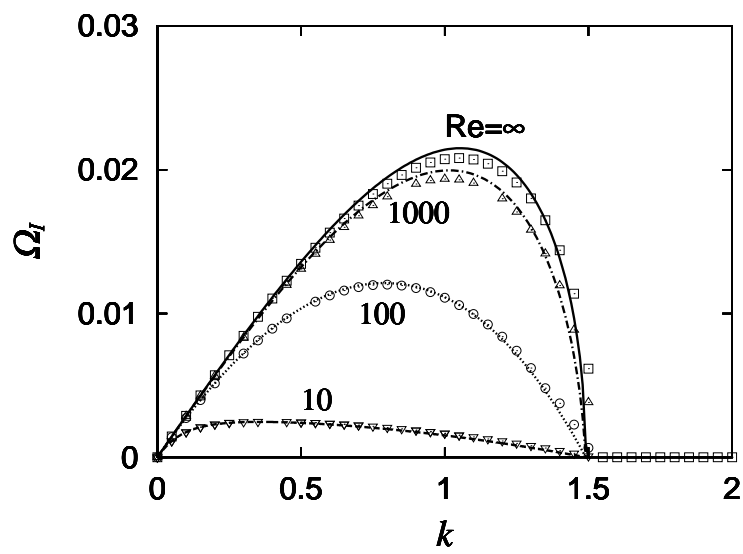

(b)

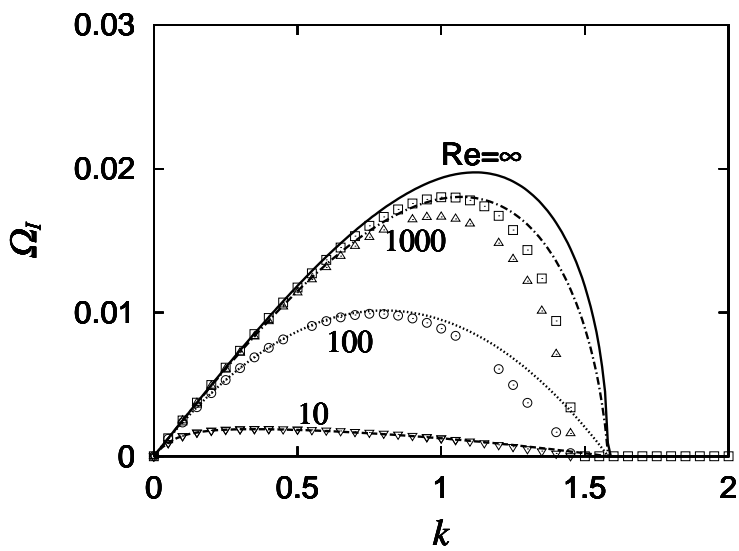

Figure 2. Comparison of the growth rates $\Omega_{I}$ between the exact instability and the instability based on the long wave approximation for $\gamma=0.001$ and $\operatorname{Re}=\infty, 1000,100$ and 10 when $\mathrm{Wb}=1000$ for $H=2$ in (a) and $H=3$ in (b).

long wave approximation. Figure 2 shows typical results of the instabilities for rather large $\mathrm{Wb}$ when $\mathrm{Wb}=1000, \gamma=0.001$ and $\mathrm{Re}=\infty, 1000,100,10$, where for $H=2$ in (a) and $H=3$ in (b). In the figure the solid lines denote the long wave approximation, while the symbols denote exact solutions of the instability, where $\square, \Delta, \bigcirc$ and $\nabla$ correspond to $\operatorname{Re}=\infty, 1000,100$ and 10, respectively. It is found for $H=2$ in Fig. 2(a) that the maximum growth rate in the exact solution becomes smaller than that in the approximation as Re increases, though the cut-off wave number is almost the same in both exact and approximation for all Re. However, when $H=3$ in Fig. 2(b), the discrepancy between the exact and approximation appears not only in the maximum growth rates but also in the cut-off wave numbers, though the cut-off wave number is still independent of Re. In particular, the discrepancy of maximum growth rate between the exact and the approximation increases with the increase of $H$ as well as Re.

We note that the discrepancy between both results is obvious for $H \gtrsim 3$ when $\mathrm{Wb}=1000$, though the approximation agrees well with the exact solution even for much larger $H$ when lower $\mathrm{Wb}$. As a result, there exists a critical length $H_{c}$ below which the present long wave approximation is valid and this $H_{c}$ decreases with the increase of $\mathrm{Wb}$. On the other hand, it is found that there exist $H_{w}$ above which the temporal growth rate $\Omega_{I m}$, the corresponding wave number $k_{m}$ and the cut-off wave number $k_{c}$ in the exact solutions are almost independent of $H$. This means that the influence of the sheath wall can be ignored in such a region as $H>H_{w}$ even if the sheath wall is considered in the analysis. Variations of these $H_{c}$ and $H_{w}$ with $\mathrm{Wb}$ are shown in Fig. 3 for $\gamma=0.0001$ (thin lines), 0.001 (medium lines) and 0.01 (thick lines), where solid lines denote for $H_{c}$ and broken lines for $H_{w}$ for the most dangerous case $\operatorname{Re}=\infty$.

It is found for any $\gamma$ that $H_{c}$ gradually decreases with the increase of $\mathrm{Wb}$ and the present long wave approximation is valid in the region $1<H<H_{c}$. In the above, $H_{c}$ is determined in such a way that the discrepancy of $k_{c}$ between the exact and approximation is relatively within $5 \%$ tolerance. On the other hand, it is found that there exist $\mathrm{Wb}$ that $H_{w}$ takes the largest value for each $\gamma$ and this Wb increases with the decrease of $\gamma$. In the above, $H_{w}$ is determined in such a way that the relative difference of $k_{c}$ between the exact solutions at $H=H_{w}$ and $H=\infty$ is at most $5 \%$ tolerance. As a result, we can see that there exist such parameter region $H_{w}<H<H_{c}$ that the approximation is valid and the influence of the sheath wall is ignored. We note that these regions of $H_{c}$ and $H_{w}$ are determined based on not $k_{m}$ but $k_{c}$ because the value of $k_{c}$ is independent of Re as long as $H$ and $\mathrm{Wb}$ are fixed.

\section{Numerical results}

In the numerical procedure under the spatially periodic boundary condition for the time evolution equations (3) to (6), the 4th order Runge-Kutta method is used for the time derivatives and the 


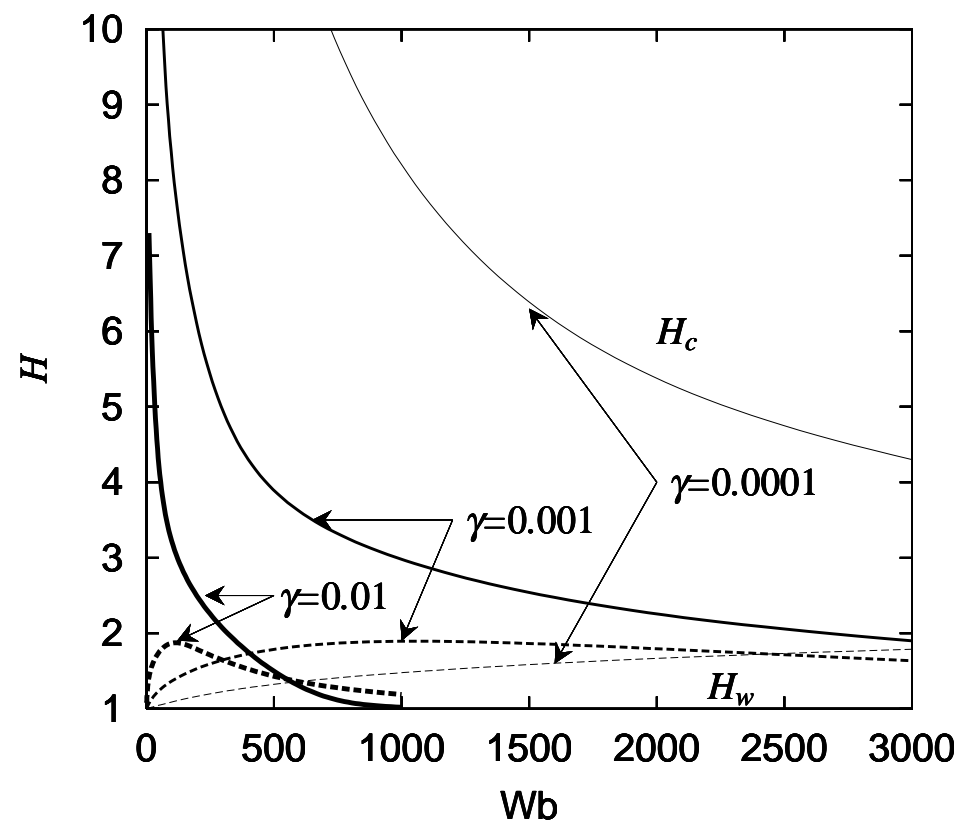

Figure 3. Validity and limitation of the approximation and influence of the sheath wall in the parameter region of $\mathrm{Wb}$ and $H$ when $\gamma=0.0001$ (thin lines), 0.001 (medium lines) and 0.01 (thick lines). The wall does not affect for $H>H_{w}$ (broken lines) and the approximation is valid for $1<H<H_{c}$ (solid lines) and, so that, the influence of the wall can be neglected for $H_{w}<H<H_{c}$ within the validity of the approximation.

finite difference (central difference) method is used for the spatial derivatives where the 3rd order up-wind scheme is adopted for the advection terms. On the other hand, the pressure equations (7) are solved by the Gauss elimination method after spatially discretizing. In these calculations, the numerical time step size $\Delta t$ is taken as 0.001 and the spatial step size $\Delta x$ is as $2 \pi /(k N)$. The wave number $k$ is taken as $k_{m}$ giving the maximum growth rate $\Omega_{I m}$ which is obtained from the dispersion relation under the long wave approximation. The number $N$ is taken as 80 in all of the calculations, where grid independency is confirmed for $N=80,120$ and 160 except a small phase shift. The precision of the present calculations is within the tolerance of $0.5 \%$ relative errors in volume ratio under the spatially periodic boundary condition at $x$ and $x+2 \pi / k_{m}$.

It is noted in the present calculations that the variables $h, w_{1}, w_{2}$ and $u_{2}$ at $t+\Delta t$ should be obtained from those variables at $t$ and the pressers $p_{2}^{(0)}$ and $p_{2}^{(1)}$ at $t+\Delta t$, while the pressures $p_{2}^{(0)}$ and $p_{2}^{(1)}$ at $t+\Delta t$ should be obtained from $h, w_{1}, w_{2}$ and $u_{2}$ at $t+\Delta t$. In order to obtain more precise solutions at $t+\Delta t$, the following procedure is adopted: First, the new $h, w_{1}, w_{2}$ and $u_{2}$ at $t+\Delta t$ are obtained by old $h, w_{1}, w_{2}, u_{2}, p_{2}^{(0)}$ and $p_{2}^{(1)}$ at $t$. Next, by using thus obtained new $h, w_{1}, w_{2}$ and $u_{2}$ at $t+\Delta t$, the new pressures $p_{2}^{(0)}$ and $p_{2}^{(1)}$ at $t+\Delta t$ are obtained. By replacing the old pressures in the first step by the new pressures in the second step, the above first and second steps are repeated until the new pressures are left unchanged, where the convergence is achieved when the relative error is less than $10^{-8}$.

In the following, the initial condition is given as $h=1+0.01 \cos k x$ for $k=k_{m}$ and $w_{1}=1$, $u_{1}=0, w_{2}=u_{2}=0, p_{1}=1 / \mathrm{Wb}$ and $p_{2}^{(0)}=p_{2}^{(1)}=0$ in all of the calculations. The calculations for such initial values are carried out until the radius of the jet becomes less than 0.01 at the neck of pinching or the jet surface touches the wall within the gap length 0.01 .

We first consider the influence of $\mathrm{Wb}$ on the behavior of breakup of the jet. Figure 4 shows typical breakup profiles and time evolutions of the maximum and minimum radii when $\mathrm{Wb}=100$, $\gamma=0.001$ and $\operatorname{Re}=\infty$, where $H=1.8\left(k_{m}=0.745\right)$ in $(\mathrm{a}), H=2\left(k_{m}=0.738\right)$ in (b) and $H=3$ $\left(k_{m}=0.734\right)$ in (c), and the broken lines denote the linear theory in the long wave approximation for comparison. It is found that the breakup profiles in both (b) and (c) appear by pinching and produce large round blobs with satellite drops. However, in (a) for $H<2$, the pinching breakup 
(a)
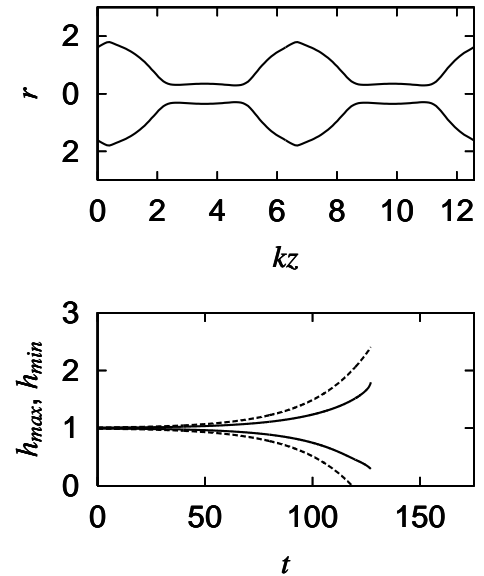

(b)
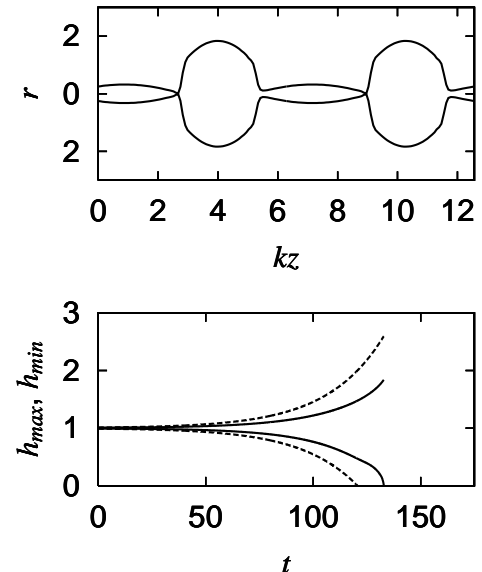

(c)
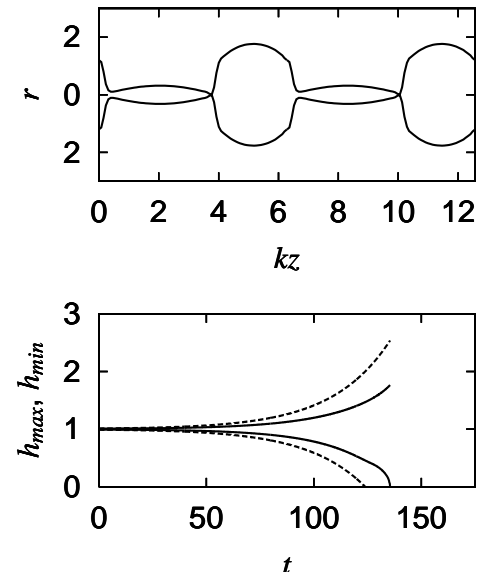

Figure 4. Profiles of the jet at the breakup and time evolutions of the maximum and minimum of $h$ when $\mathrm{Wb}=100$, $\gamma=0.001$ and $\operatorname{Re}=\infty$, where $H=1.8\left(k_{m}=0.745\right)$ in $(\mathrm{a}), H=2\left(k_{m}=0.738\right)$ in (b) and $H=3\left(k_{m}=0.734\right)$ in (c), and broken lines denote the linear results for comparison.

(a)
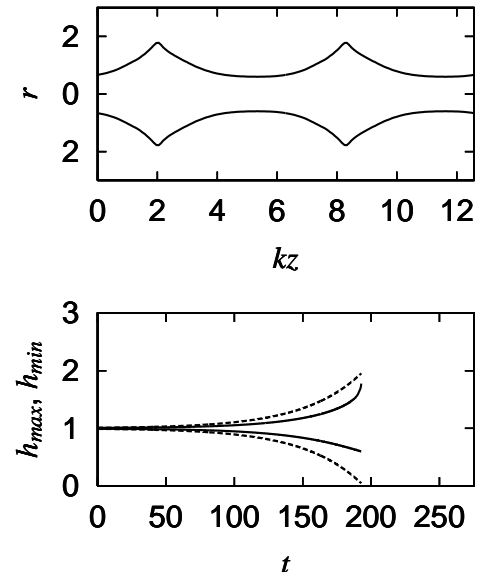

(b)
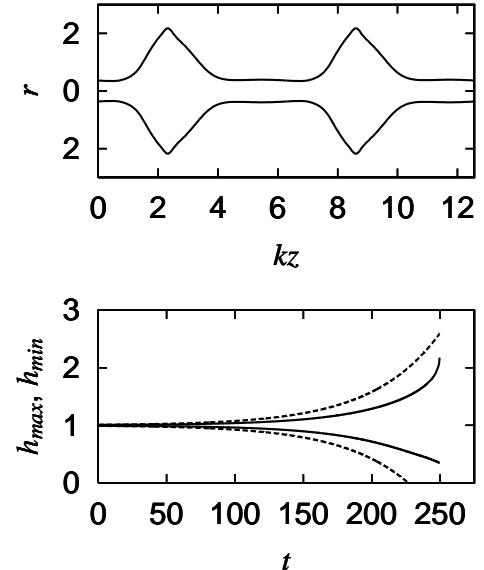

(c)
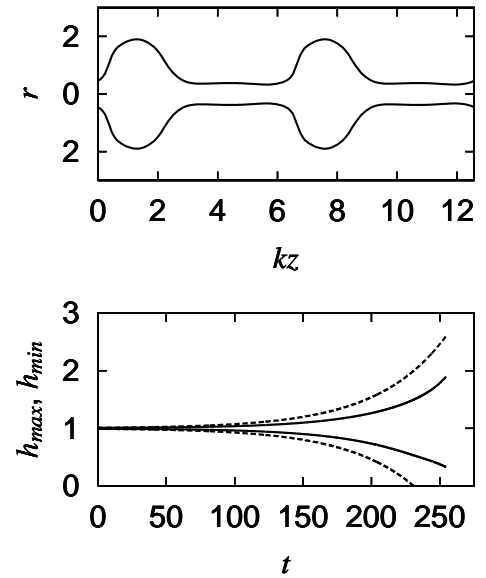

Figure 5. Profiles of the jet at the breakup and time evolutions of maximum and minimum of $h$ when $\mathrm{Wb}=1000$, $\gamma=0.001$ and $\operatorname{Re}=\infty$, where $H=1.8\left(k_{m}=1.087\right)$ in (a), $H=2.2\left(k_{m}=1.042\right)$ in (b) and $H=2.3\left(k_{m}=1.042\right)$ in (c), and broken lines denote the linear results for comparison.

does not appear and instead small tips appear at the top and down of the blobs. It is also found that the time evolutions of the increase and decrease of radii are always overestimated by the linear theory. In the nonlinear theory, the minimum radii decrease rapidly in the final stage in (b) and (c) which leads to the breakup by pinching, while in (a) the maximum radius increases more than the decrease of the minimum one in the final stage.

For much larger $\mathrm{Wb}$, the breakup properties drastically change. Figure 5 shows breakup profiles and time evolutions of the maximum and minimum radii when $\mathrm{Wb}=1000, \gamma=0.001$ and Re $=\infty$, where $H=1.8\left(k_{m}=1.087\right)$ in (a), $H=2.2\left(k_{m}=1.042\right)$ in (b) and $H=2.3$ $\left(k_{m}=1.042\right)$ in (c), and the broken lines denote the linear theory for comparison. It is found that a spiked profile like a 'cusp' and a moderate trough appear in (a) and (b), while the structure of round blobs connected by threads appears in (c) when $H$ slightly increases. In the time evolutions, the maximum radius drastically increases in the final stage in (a) and (b), while both maximum and minimum radii vary almost at the same rate in (c), where the linear theory still over predicts the increase and decrease of $h$.

As for the classification of these breakup modes, we have seen that in the Rayleigh mode the minimum radius rapidly decreases near the breakup and large liquid drops are produced by pinching, while in the second wind-induced mode the maximum radius rapidly increase near 
(a)

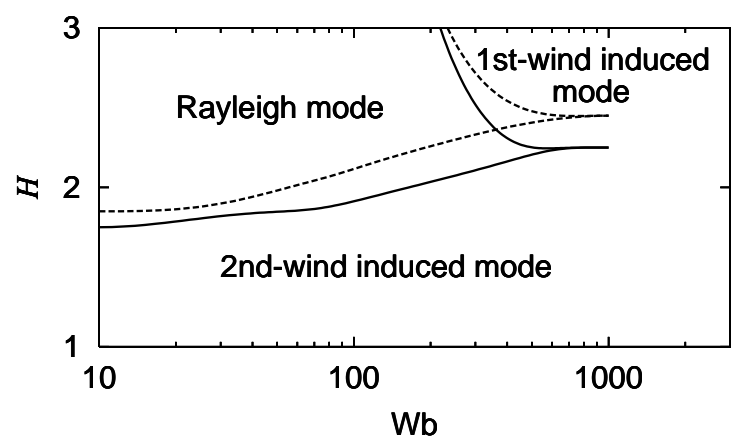

(b)

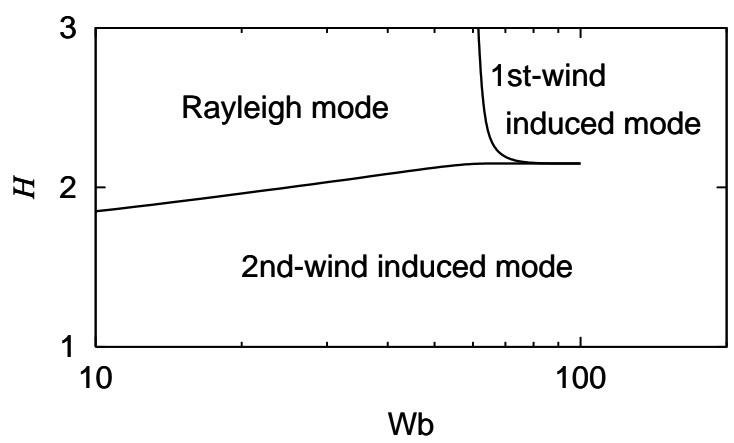

Figure 6. Regions of the Rayleigh and Taylor (1st- and 2nd-wind induced) modes in the parameter space of Wb and $H$, where $\gamma=0.001$ for $\operatorname{Re}=\infty$ (solid lines) and 100 (broken lines) in (a), while $\gamma=0.01$ for $\operatorname{Re}=\infty$ in (b).

the breakup and cusp profiles appear. In the first wind-induced mode, however, variation rates of the maximum and minimum radii are almost the same and blobs are connected by threads without pinching, though finally the long thread would be broken up due to the instability.

According to these criteria, we examine existing regions of the Rayleigh, first and second windinduced modes more precisely in the parameter space of $\mathrm{Wb}$ and $H$ for different $\gamma$ and Re. Figure 6 shows the regions of these modes in the parameter space for $10 \leq \mathrm{Wb} \leq 1000$ and $1 \leq H \leq 3$, where the cases of $\operatorname{Re}=\infty$ (solid lines) and 100 (broken lines) for $\gamma=0.001$ are shown in (a), while $\operatorname{Re}=\infty$ for $\gamma=0.01$ in (b). It is found in Fig. 6(a) and (b) that the second wind-induced mode prevails always for smaller $H$ whose range gradually increases with the increase of $\mathrm{Wb}$. The range of $H$ in the second wind-induced mode increases as Re decreases and $\gamma$ increases.

On the other hand, when $H$ increases over the above range, the Rayleigh mode prevails for smaller $\mathrm{Wb}$ and the first wind-induced mode for larger $\mathrm{Wb}$. The increase of Re and $\gamma$ diminish the range of $\mathrm{Wb}$ for the Rayleigh mode. It is also found in Fig. $6(\mathrm{a})$ for $200 \lesssim \mathrm{Wb} \lesssim 500$ that the Rayleigh mode appearers between the second and first wind-induced modes in $H$. The above Rayleigh mode between the first and second wind-induced modes is also seen in Fig. 6(b) for $60 \lesssim \mathrm{Wb} \lesssim 80$.

As is shown in Fig. 3, the wall effect may appear for $1<H<H_{w}$ within the $5 \%$ torelance in the linear regime. However, as is seen in the breakup profiles, this is not always the case in the nonlinear regime. In fact, according to Fig. 3, the wall effect may appear for $H_{w}=4.3$ when $\mathrm{Wb}=100$ and $\gamma=0.001$. However, Fig. 4 shows that the breakup profiles for $H=2$ and 3 are almost the same except for some phase shift, while the breakup profiles for $H=1.8$ becomes completely different. This means that $1.8<H_{w}<2$ in the nonlinear regime, which can not be expected from the linear analysis. For large Weber number, the wall effect may appear for $H_{w}=2.8$ when $\mathrm{Wb}=1000$ and $\gamma=0.001$ in the linear regime, while Fig. 5 shows that $2.2<H_{w}<2.3$ in the nonlinear analysis. Resulting from this, the border for appearance of the wall effect is shifted to lower $H$ in the nonlinear results.

\section{Concluding remarks}

The instabilities and breakup phenomena of a liquid column jet in a stationary surrounding gas have been analytically investigated, when the jet flows through a coaxial cylindrical sheath.

Validity and limitation of the long wave approximation are examined in comparison with the exact linear instabilities. It is found that there exist two critical sheath radii $H_{c}$ and $H_{w}$, where the present approximation is valid when $H<H_{c}$, while the influence of the wall disappears when $H>H_{w}$. Therefore, for $H_{w}<H<H_{c}$ the approximation is valid and the influence of the wall can be neglected. However, it is seen from the breakup profiles that $H_{w}$ in the nonlinear regime is more reduced than the linear expectation as $\mathrm{Wb}$ and $\gamma$ decrease.

On the other hand, it is found that there exist three types of breakup profiles whose breakup 
modes are called the Rayleigh and first and second wind-induced modes. The existing regions of these modes are examined in the parameter space of $\mathrm{Wb}$ and $H$, which is determined from geometrical properties in the breakup profiles and rapid increase or decreases of $h$ in the final stage. Critical curves to distinguish these modes in the parameter space for different $\gamma$ and $\operatorname{Re}$ are obtained. Then the mode transition of the second wind-induced mode to the Rayleigh or to the first wind-induced mode occurs generally when $H$ increases, while the transition of the Rayleigh mode to the first wind-induced mode appears when Wb increases.

It is shown experimentally [9] for the unbounded surrounding gas that the first-wind induced mode appears when $600 \lesssim \mathrm{Wb} \lesssim 6500$ for $\gamma=0.001$, while the second-wind induced mode appears when $6500 \lesssim \mathrm{Wb} \lesssim 20000$ for $\gamma=0.001$. Although these experimental results relatively agree well with the numerical results by Spangler et al. [14], the present results for $10 \leq \mathrm{Wb} \leq$ 1000 and $\gamma=0.001$ show that the first wind-induced modes can appear for larger Wb than $200 \sim$ 500 depending on $H$, while the second wind induced mode appears when $H \lesssim 2$ depending on $\mathrm{Wb}$. Consequently, the coaxial sheath wall is available to produce fine droplets resulting from the second wind-induced mode even in much smaller $\mathrm{Wb}$ by placing the sheath wall closely to the jet.

\section{Acknowledgements}

This work has been partially supported by JSPS KAKENHI Grant Number JP16K06082.

\section{References}

[1] Lefebvre A H 1989 Atomization and sprays (Hemisphare Publishing Corporation)

[2] Eggers J 1997 Nonlinear dynamics and breakup of free surface flows Rev. Mod. Phys. 69 865-929

[3] Lin S P 2003 Breakup of liquid sheets and jets (Cambridge Univ. Press)

[4] Eggers J and Villermaux E 2008 Physics of liquid jets Rep. Prog. Phys. 71036601

[5] Ashgriz N and Yarin A L 2011 Capillary instability of free liquid jets Handbook of Atomization and Spray ed N Ashgriz (Springer) 20-35

[6] Lord Rayleigh 1879 On the instability of jets Proc. London Math. Soc. 10 4-13

[7] Grant R P and Middleman S 1966 Newtonian jet stability A. I. Ch. E. J. 2 669-678

[8] Reitz R D and Bracco F V 1982 Mechanism of atomization of a liquid jet Phys. Fluids 25 1730-1742

[9] Chigier N and Reitz R D 1996 Regimes of jet breakup and breakup mechanism (In Recent Advances in Spray Combustion: Spray Atomization and Drop Burning Phenomena vol 1) ed K K Kuo AIAA 109-135

[10] Lin S P and Reitz R D 1998 Drop and spray formation from a liquid jet Ann. Rev. Fluid Mech. 30 85-105

[11] Yang H Q 1992 Asymmetric instability of a liquid jet Phys. Fluids A 4 681-689

[12] Avital A 1995 Asymmetric instability of a viscid capillary jet in an inviscid media Phys. Fluids 7 1162-1164

[13] Ruo A-C, Chang M-H and Chen F 2008 On the nonaxisymmetric instability of round liquid jet Phys. Fluids 20062105

[14] Spangler C A, Hibling J H and Heister S D 1995 Nonlinear modeling of jet atomization in the wind-induced regime Phys. Fluids 7 964-971

[15] Yoshinaga T 2020 Instabilities of a liquid column jet in a surrounding gas flowing through a coaxial cylindrical sheath Fluid Dyn. Res. 52045502 